\title{
Regional myocardial function at preclinical disease stage of hypertrophic cardiomyopathy in female gene variant carriers
}

\author{
Rahana Y. Parbhudayal ${ }^{1,2,3} \cdot$ Celine Seegers $^{1} \cdot$ Pierre Croisille $^{4} \cdot$ Patrick Clarysse $^{4} \cdot$ Albert C. van Rossum $^{1,3}$. \\ Tjeerd Germans ${ }^{1} \cdot$ Jolanda van der Velden ${ }^{2,3}$
}

Received: 18 August 2020 / Accepted: 31 December 2020 / Published online: 9 February 2021

(c) The Author(s) 2021

\begin{abstract}
We recently showed more severe diastolic dysfunction at the time of myectomy in female compared to male patients with obstructive hypertrophic cardiomyopathy. Early recognition of aberrant cardiac contracility using cardiovascular magnetic resonance (CMR) imaging may identify women at risk of cardiac dysfunction. To define myocardial function at an early disease stage, we studied regional cardiac function using CMR imaging with tissue tagging in asymptomatic female gene variant carriers. CMR imaging with tissue tagging was done in 13 MYBPC3, 11 MYH7 and 6 TNNT2 gene carriers and 16 age-matched controls. Regional peak circumferential strain was derived from tissue tagging images of the basal and midventricular segments of the septum and lateral wall. Left ventricular wall thickness and global function were comparable between MYBPC3, MYH7, TNNT2 carriers and controls. MYH7 gene variant carriers showed a different strain pattern as compared to the other groups, with higher septal peak circumferential strain at the basal segments compared to the lateral wall, whereas $M Y B P C 3, T N N T 2$ carriers and controls showed higher strain at the lateral wall compared to the septum. Only subtle gene-specific changes in strain pattern occur in the myocardium preceding development of cardiac hypertrophy. Overall, our study shows that there are no major contractile deficits in asymptomatic females carrying a pathogenic gene variant, which would justify the use of CMR imaging for earlier diagnosis.
\end{abstract}

Keywords Hypertrophic cardiomyopathy $\cdot$ Tissue tagging $\cdot$ MYBPC3 $\cdot$ MYH7 $\cdot$ TNNT2

\section{Abbreviations \\ CMR Cardiovascular magnetic resonance \\ HCM Hypertrophic cardiomyopathy \\ LVEF Left ventricular ejection fraction \\ LVH Left ventricular hypertrophy \\ $M Y B P C 3 \quad$ Myosin binding protein-C gene}

\section{Supplementary Information The online version of this article} (https://doi.org/10.1007/s10554-020-02156-1) contains

supplementary material, which is available to authorized users.

Jolanda van der Velden

j.vandervelden1@amsterdamumc.nl

1 Department of Cardiology, Amsterdam UMC, Amsterdam Cardiovascular Sciences, Vrije Universiteit Amsterdam, Amsterdam, The Netherlands

2 Department of Physiology, Amsterdam UMC, Amsterdam Cardiovascular Sciences, Vrije Universiteit Amsterdam, De Boelelaan 1117, 1081 HV Amsterdam, The Netherlands

3 The Netherlands Heart Institute, Utrecht, The Netherlands

4 Univ Lyon, UJM-Saint-Etienne, INSA, CNRS UMR 5520, INSERM U1206, CREATIS, 42023 Saint-Etienne, France
MYH7 $\quad \beta$-Myosin heavy chain gene

TNNT2 Troponin T gene

\section{Introduction}

Hypertrophic cardiomyopathy (HCM) is the most common genetic cardiomyopathy with an autosomal dominant pattern of inheritance [1]. HCM typically presents with asymmetric left ventricular hypertrophy (LVH) most frequently at the basal septum, in the absence of any abnormal loading conditions [2]. A causative gene variant (i.e. mutation) in genes encoding sarcomere proteins is identified in approximately $50-60 \%$ of all index patients (genotype-positive individuals) [3]. In the majority of patients, variants in the genes encoding thick filament proteins myosin binding protein-C (MYBPC3) and $\beta$-myosin heavy chain (MYH7) and the thin filament protein troponin $\mathrm{T}$ (TNNT2) are found [4]. We recently observed more severe diastolic dysfunction in female compared to male patients with obstructive hypertrophic cardiomyopathy at the time of cardiac surgery 
[5]. Correction of cardiac dimensions by body surface area (BSA) revealed more severe cardiac remodeling in female compared to male patients evident from a significantly higher BSA-indexed left atrial dimension and BSAindexed septal thickness. A subsequent study in a cohort of genotype-positive subjects referred for family screening indicated that correcting maximal wall thickness for body size and applying specific cut-off values improved the predictive accuracy for HCM-related events [6]. These recent studies indicate that females may be underrepresented in HCM patient studies because of the current HCM diagnostic criterium of $\geq 15 \mathrm{~mm} \mathrm{LV}$ wall thickness $(\geq 13 \mathrm{~mm}$ in case of first-degree family members) [2], which does not take into account body size [7]. Indeed, the percentage of female patients in HCM patient cohort studies is on average $30-40 \%$ [8-10], which may be explained by lower disease penetrance, but could also imply that cardiac dysfunction remains undetected, in particular in the female HCM patient group, using cardiac remodeling, i.e. hypertrophy, rather than cardiac dysfunction as diagnostic criterium.

To detect early gene variant-related functional changes in in vivo cardiac function, studies are warranted in asymptomatic gene variant carriers without cardiac remodeling (i.e. no hypertrophy, no fibrosis, no capillary rarefaction) using advanced cardiac imaging. Here, we used cardiovascular magnetic resonance (CMR) imaging with high resolution tissue tagging to investigate if regional myocardial functional differences exist in female asymptomatic gene variant carriers who harbor common HCM gene variants in thick (MYBPC3, MYH7) and thin (TNNT2) filament genes.

\section{Material and methods}

The CMR imaging studies in this study included 30 female asymptomatic carriers with gene variants in $M Y B P C 3$ $(\mathrm{n}=13)$, MYH7 $(\mathrm{n}=11)$ and TNNT2 $(\mathrm{n}=6)$. Gene variant carriers were included after genetic screening, classified as likely pathogenic and pathogenic (clinically graded class 4 or 5), and were first-degree relatives of index HCM patients. All gene variant carriers had a wall thickness of the $\mathrm{LV}<13 \mathrm{~mm}$ (based on ESC guidelines) and were free of any systemic and/or cardiac disease and used no medication. Data from gene variant carriers were compared with data from 16 healthy female controls, who were age and gender matched. Out of the 46 study participants 4 controls, 2 MYBPC 3 gene variant carriers, $4 M Y H 7$ gene variant carriers and 2 TNNT2 gene variant carriers were interrelated. All participants underwent a CMR imaging protocol. The study conformed to the principles outlined in the Declaration of Helsinki and was approved by the Medical Ethical Committee of the VU University Medical Center Amsterdam. Written informed consent was obtained from all study participants. The STROBE checklist has been used for preparing the manuscript.

\section{Cardiovascular magnetic resonance imaging}

CMR imaging was performed using a $1.5 \mathrm{~T}$ whole body scanner (Avanto, Siemens, Erlangen, Germany), with a sixchannel phased-array body coil. A stack of short axis cines was used for LV full coverage. Cine images were acquired in a single breath-hold using a balanced segmented steady-state free precession (SSFP) [11]. Also, 4, 3 and 2 chamber long axis SSFP cine images were obtained. From the short axis cine images LV end-diastolic and end-systolic volumes and mass were obtained. Typical image parameters were: $5 \mathrm{~mm}$ slice thickness with $5 \mathrm{~mm}$ gap between short-axis slices, temporal resolution $<50 \mathrm{~ms}$, repetition time $3.2 \mathrm{~ms}$, echo time $1.54 \mathrm{~ms}$, flip angle $60^{\circ}$, and typical image resolution $1.3 \times 1.6 \mathrm{~mm}$.

For regional function assessment, myocardial tissue tagging imaging was performed using a multiple breath-hold, retrospectively triggered SSFP myocardial tissue tagging sequence with the linearly increasing start-up approach [12]. See Fig. 1. Two short axis planes were positioned at 25 and 50 percent of the distance between the mitral valve annulus and the apex (Fig. 2a). Image parameters were: $7 \mathrm{~mm}$ slice thickness, temporal resolution $14 \mathrm{~ms}$, repetition time $4.7 \mathrm{~ms}$, echo time $2.3 \mathrm{~ms}$, flip angle $20^{\circ}$, and in-plane image resolution of 1.2 by $3.8 \mathrm{~mm}$, with $7 \mathrm{~mm}$ tag spacing.

Late Gadolinium enhancement images were obtained $10 \mathrm{~min}$ after injection of $0.2 \mathrm{mmol} / \mathrm{kg}$ Gadolinium-DTPA. An inversion recovery Fast low angle shot sequence was used to obtain images with $6 \mathrm{~mm}$ slice thickness planned in the same orientation as the long and short axis cines.

\section{Post processing}

LV volumes and mass analysis were performed by a single investigator, using Circle CVi42, Calgary, Canada. Endocardial contours were drawn to calculate LV end-diastolic (LVEDV) and end-systolic volumes (LVESV) and ejection fraction (LVEF). Epicardial contours were added to calculate LV end-diastolic wall thickness and LV mass. Papillary muscles were included in LV volumes and excluded from LV mass. LV end-diastolic, end-systolic volumes and LV mass were indexed for body surface area. End-diastolic wall thickness at the septum and lateral wall were derived from respectively the mean of four septal (anteroseptal and inferoseptal) and lateral segments (anterolateral and inferolateral) at the basal and midventricular level.

Circumferential strain analysis was obtained from the $50 \%$ mid myocardial layer from tissue tagging cines (Fig. 2b), using Intag software (CREATIS, Lyon, France), and has been reported to be most reproducible $[13,14]$. 


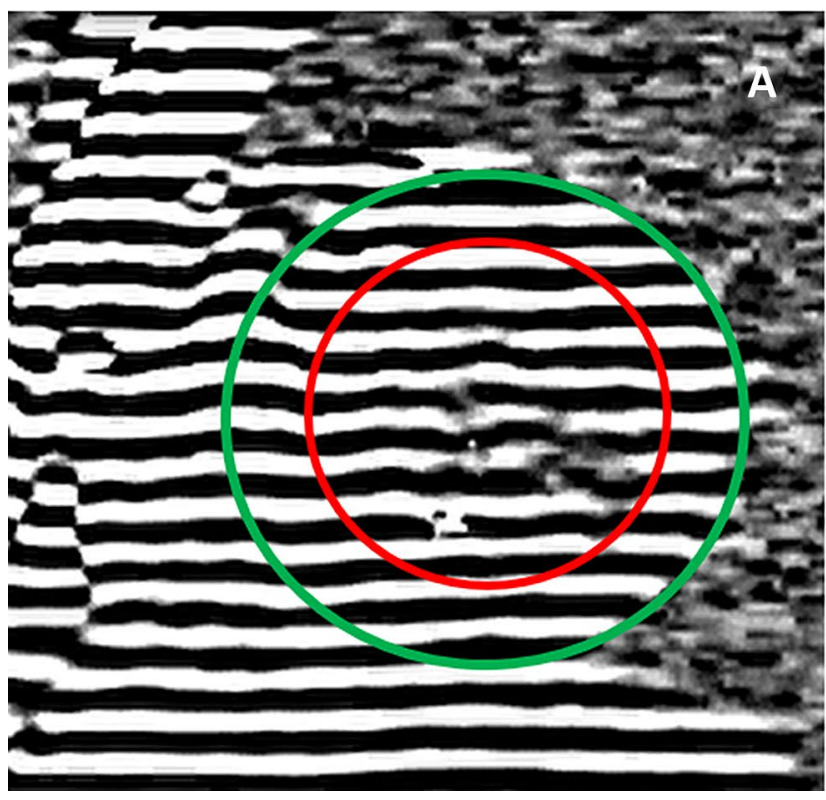

Fig. 1 SSFP Myocardial tissue tagging. a At end-diastole, a line tagging grid is applied. The myocardium is delineated by the epicardial (green circle) and endocardial (red circle) contours. b As the taglines

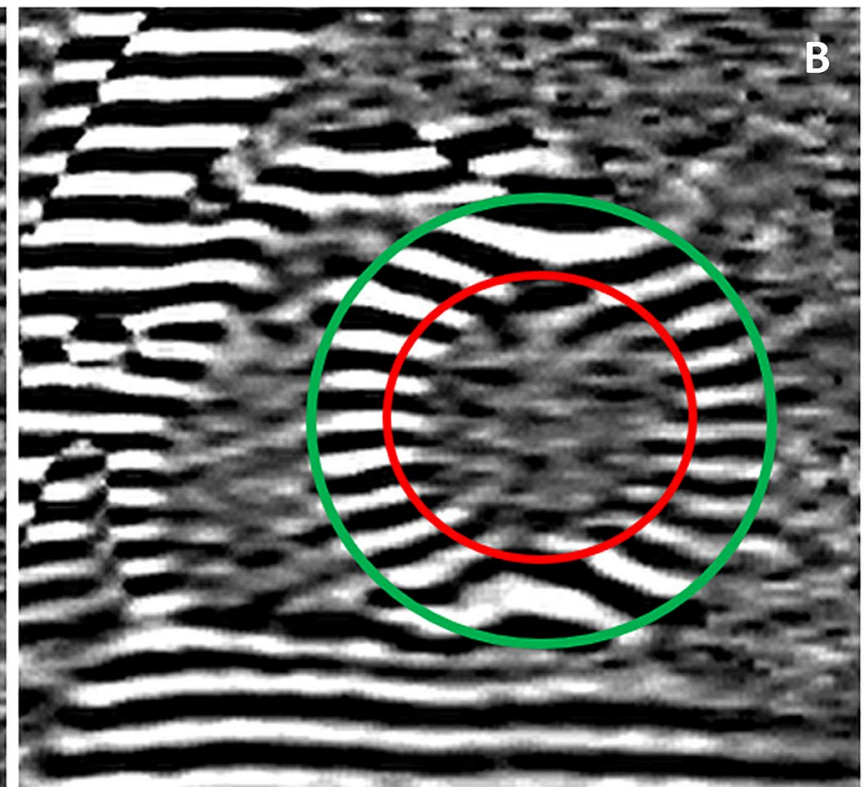

are a temporary property of the myocardium, deformation (strain) can be depicted and quantified by this method, as illustrated by this endsystolic image
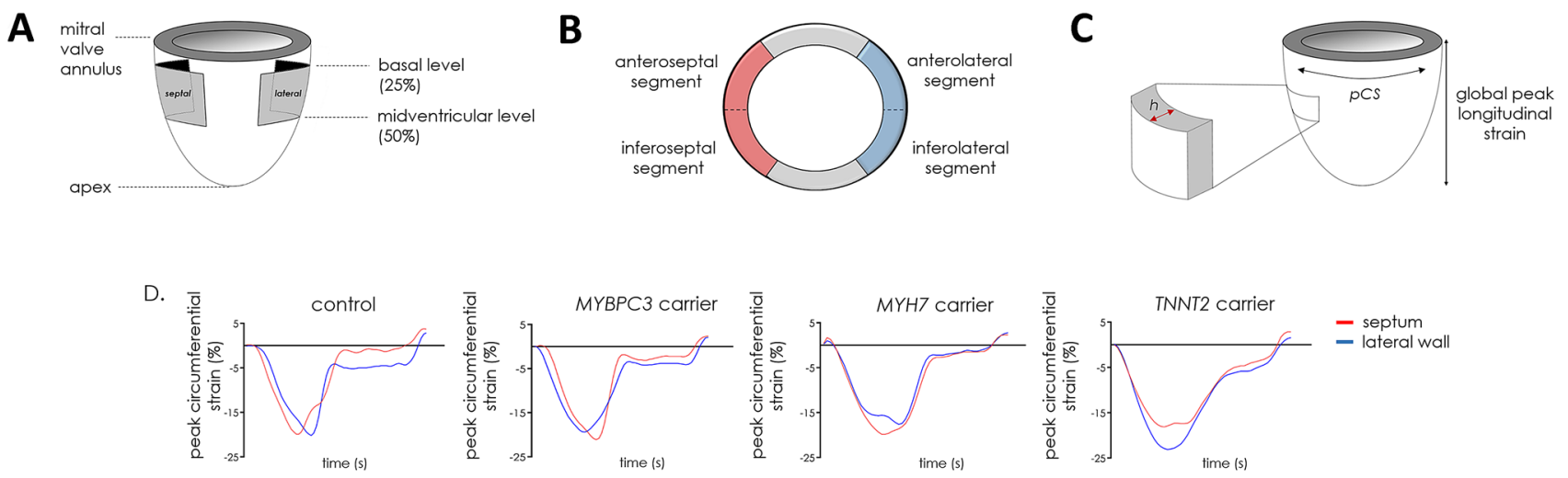

Fig. 2 Long and short axis images of the left ventricle. a Schematic image of a long axis of the left ventricle. Depicted are the two positions of the basal (25\%) and midventricular (50\%) levels where myocardial tissue tagging was applied. b Four septal segments (two anteroseptal and two inferoseptal) were compared to the four lateral segments (two anterolateral and two inferolateral) at basal and midventricular level c Schematic image of global peak longitudinal

LV segmentation was performed according to the $17 \mathrm{seg}$ ment AHA model [15]. From this analysis, peak circumferential strain and peak diastolic circumferential strain rate per segment were obtained. The four septal segments (at basal and midventricular level antero- and inferoseptal segments) were compared with the four lateral segments (at basal and midventricular level antero- and inferolateral segments) (Fig. 2b). Representative peak circumferential strain. At basal and midventricular level, end-diastolic wall thickness (h) and peak circumferential strain (pCS) were measured according to the 17 segment AHA classification. $\mathbf{d}$ Representative peak circumferential strain curves of one healthy control subject, one $M Y B P C 3$, one $M Y H 7$ and one TNNT2 gene variant carrier are shown. The red curves indicate the septum. The blue curves indicate the lateral wall

strain curves of 1 healthy control subject and 3 gene variant carriers are shown in Fig. 2d (red curves indicate the septum and blue curves indicate the lateral wall). Global longitudinal strain was obtained from the 4, 3 and 2 chamber long axis cines with tissue tracking using CVi 42 software (Circle Cardiovascular Imaging, Calgary, Canada) (Fig. 2c). 


\section{Statistical analysis}

Statistical analysis was performed using SPSS software (version 22.0; SPSS, Chicago, IL, USA). Normality of data was inspected visually by means of QQ-plots. Means of continuous demographic and outcome variables were compared between gene variant carrier groups using ANOVA with a Bonferroni post-hoc analysis after normality was verified. Exact chi-square test was used for categorical demographic variables. A mixed model analyses was used to test whether regional differences in mean wall thickness and peak circumferential strain differed between gene variant carriers groups and controls. The model included fixed effects for gene variant group, region (septal or lateral) and their twoway interaction and a random effect for subject. In case of a significant two-way interaction, post-hoc analysis with Bonferroni correction were performed to test for regional differences within each gene variant carrier and control group separately. As two separate statistical tests were performed for basal and midventricular segments a two-sided significance level of $0.05 / 2$ was used for all statistical tests to account for multiple testing. For baseline characteristics a significance level of $<0.05$ was used.

\section{Results}

Table 1 summarizes genetic and clinical parameters of all study participants. Overall, controls and carriers were of similar age and had similar BSA. No differences were present in cardiac function (ejection fraction, stroke volume) and left ventricular mass between controls and carrier groups. LVEDV and LVESV in TNNT2 group were significantly smaller than in $M Y B P C 3$, but similar to controls and $M Y H 7$ (Table 1). None of the gene variant carriers and controls showed contrast enhanced myocardial areas.

\section{Regional anatomical parameters}

End-diastolic wall thickness of basal and midventricular segments of the septum and lateral wall were comparable between MYBPC3, MYH7 and TNNT2 groups and controls (Table 2). In addition, septal-to-lateral wall thickness (S/L) ratio of basal and midventricular segments of the septum and lateral wall were comparable between gene variant carrier groups and controls (Table 2).

\section{Regional functional parameters}

Global longitudinal strain was similar between $M Y B P C 3$, MYH7 and TNNT2 gene variant carriers $(-21.5 \pm 2.2$, $-23.0 \pm 1.9$ and $-22.0 \pm 3.1 \%$, respectively) and controls $(-21.4 \pm 2.0 \%)$. Analysis of peak circumferential strain showed higher strain for the lateral segments compared to septal segments, both at basal and midventricular level, with significant differences between the septum and lateral segments at basal level in the MYBPC3, TNNT2 gene variant carriers and controls (Fig. 2a, Table S1). Overall, this regional (septum vs. lateral wall) difference was observed in all gene variant carrier groups and controls, except for the $M Y H 7$ gene variant group at basal level which showed an opposite pattern with a higher strain in the septum than in the lateral segments (Fig. 3a, Table S1). The difference in peak circumferential strain between septal and lateral segments was calculated per individual and is depicted in Fig. 3b. The delta (difference between septum and lateral wall) is similar in all groups, except for the $M Y H 7$ gene variant group at basal level. The value in the $M Y H 7$ gene variant group is significantly different from the value observed in the $M Y B P C 3$ group and controls (Fig. 3b). Peak diastolic circumferential strain rate of the basal and midventricular segments of the septum and lateral wall were comparable between gene variant carrier groups and controls (Table S2).

Table 1 Demographics and left ventricular parameters

\begin{tabular}{|c|c|c|c|c|}
\hline & Controls $(n=16)$ & $M Y B P C 3$ carriers $(\mathrm{n}=13)$ & $M Y H 7$ carriers $(\mathrm{n}=11)$ & $T N N T 2$ carriers $(\mathrm{n}=6)$ \\
\hline Genotype & No genotype & c. 2373 dupG $(n=13)$ & $\begin{array}{l}\text { c. } 4130 \mathrm{C}>\mathrm{T}(n=5) \\
\text { c. } 5135 \mathrm{G}>\mathrm{A}(n=2) \\
\text { c. } 1207 \mathrm{C}>\mathrm{T}(n=3) \\
\text { c. } 1727 \mathrm{~A}>\mathrm{G}(n=1)\end{array}$ & $\begin{array}{l}\text { c. } 304 \mathrm{C}>\mathrm{T}(n=3) \\
\text { c. } 856 \mathrm{C}>\mathrm{T}(n=1) \\
\text { c. } 403 \mathrm{C}>\mathrm{T}(n=1) \\
\text { c. } 277 \mathrm{G}>\mathrm{A}(n=1)\end{array}$ \\
\hline Age & $44 \pm 12$ & $37 \pm 14$ & $38 \pm 14$ & $43 \pm 15$ \\
\hline $\operatorname{BSA}\left(\mathrm{m}^{2}\right)$ & $1.80 \pm 0.09$ & $1.73 \pm 0.11$ & $1.78 \pm 0.17$ & $1.77 \pm 0.19$ \\
\hline $\operatorname{LVEDV}\left(\mathrm{ml} \cdot \mathrm{m}^{-2}\right)$ & $75.8 \pm 10.2$ & $83.6 \pm 7.3$ & $79.6 \pm 8.9$ & $69.6 \pm 15.5^{*}$ \\
\hline $\operatorname{LVESV}\left(\mathrm{ml} \cdot \mathrm{m}^{-2}\right)$ & $25.1 \pm 4.9$ & $28.4 \pm 5.8$ & $26.2 \pm 4.4$ & $19.8 \pm 5.1 *$ \\
\hline $\mathrm{SV}\left(\mathrm{ml} \cdot \mathrm{m}^{-2}\right)$ & $50 \pm 8$ & $55 \pm 9$ & $53 \pm 8$ & $50 \pm 11$ \\
\hline $\mathrm{LV}$ mass $\left(\mathrm{g} \cdot \mathrm{m}^{-2}\right)$ & $36.8 \pm 6.5$ & $55.1 \pm 9.3$ & $53.3 \pm 7.6$ & $49.8 \pm 11.3$ \\
\hline
\end{tabular}

Data are presented as mean \pm standard deviation. MYBPC3 myosin binding protein $\mathrm{C}$ gene, $M Y H 7$ myosin heavy chain gene, TNNT2 troponin $\mathrm{T}$ gene. $B S A$ body surface area, $L V E F$ left ventricular ejection fraction, $S V$ stroke volume. ${ }^{*} p<0.05$ versus $M Y B P C 3$ 
Table 2 Regional anatomical differences between gene variant carriers and controls

\begin{tabular}{|c|c|c|c|c|}
\hline & Controls $(n=16)$ & $\begin{array}{l}M Y B P C 3 \text { carriers } \\
(\mathrm{n}=13)\end{array}$ & $\begin{array}{l}\text { MYH7 carriers } \\
(\mathrm{n}=11)\end{array}$ & $\begin{array}{l}T N N T 2 \\
\text { carriers } \\
(\mathrm{n}=6)\end{array}$ \\
\hline \multicolumn{5}{|l|}{ Basal level } \\
\hline \multicolumn{5}{|l|}{ Septum } \\
\hline EDWT (mm) & $5.9 \pm 0.5$ & $5.7 \pm 1.0$ & $5.2 \pm 0.9$ & $5.2 \pm 1.3$ \\
\hline $\operatorname{EDWT}\left(\mathrm{mm} \cdot \mathrm{m}^{-2}\right)$ & $3.3 \pm 0.4$ & $3.3 \pm 0.5$ & $2.9 \pm 0.5$ & $2.9 \pm 0.4$ \\
\hline \multicolumn{5}{|l|}{ Lateral wall } \\
\hline EDWT (mm) & $5.6 \pm 0.7$ & $5.3 \pm 0.7$ & $5.4 \pm 0.7$ & $5.5 \pm 1.2$ \\
\hline $\operatorname{EDWT}\left(\mathrm{mm} \cdot \mathrm{m}^{-2}\right)$ & $3.1 \pm 0.4$ & $3.1 \pm 0.4$ & $3.0 \pm 0.3$ & $3.1 \pm 0.6$ \\
\hline $\mathrm{S} / \mathrm{L}$ ratio & $1.17 \pm 0.1$ & $1.1 \pm 0.2$ & $1.0 \pm 0.1$ & $1.0 \pm 0.3$ \\
\hline \multicolumn{5}{|l|}{ Midventricular level } \\
\hline \multicolumn{5}{|l|}{ Septum } \\
\hline EDWT wall (mm) & $5.4 \pm 0.7$ & $5.1 \pm 0.9$ & $5.3 \pm 1.0$ & $5.5 \pm 1.3$ \\
\hline $\operatorname{EDWT}\left(\mathrm{mm} \cdot \mathrm{m}^{-2}\right)$ & $3.3 \pm 0.4$ & $3.3 \pm 0.5$ & $2.9 \pm 0.5$ & $2.9 \pm 0.4$ \\
\hline \multicolumn{5}{|l|}{ Lateral wall } \\
\hline EDWT (mm) & $4.6 \pm 0.6$ & $4.2 \pm 0.5$ & $4.3 \pm 0.6$ & $4.1 \pm 0.6$ \\
\hline $\operatorname{EDWT}\left(\mathrm{mm} \cdot \mathrm{m}^{-2}\right)$ & $3.1 \pm 0.4$ & $3.1 \pm 0.4$ & $3.0 \pm 0.3$ & $3.1 \pm 0.6$ \\
\hline $\mathrm{S} / \mathrm{L}$ ratio & $1.2 \pm 0.1$ & $1.2 \pm 0.2$ & $1.2 \pm 0.2$ & $1.2 \pm 0.1$ \\
\hline
\end{tabular}

Data are presented as mean \pm standard deviation. EDWT: end-diastolic wall thickness; S/L: septum-to-lateral wall thickness. MYBPC3: myosin binding protein $\mathrm{C}$ gene; $M Y H 7$ : myosin heavy chain gene; TNNT2: troponin $\mathrm{T}$ gene. None of the comparisons reached significance
A

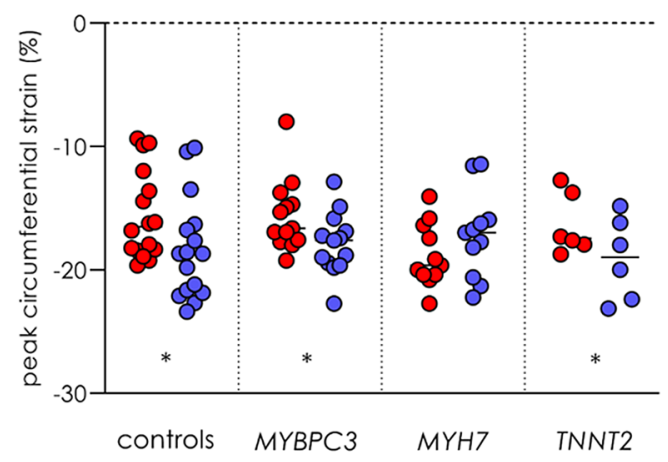

B

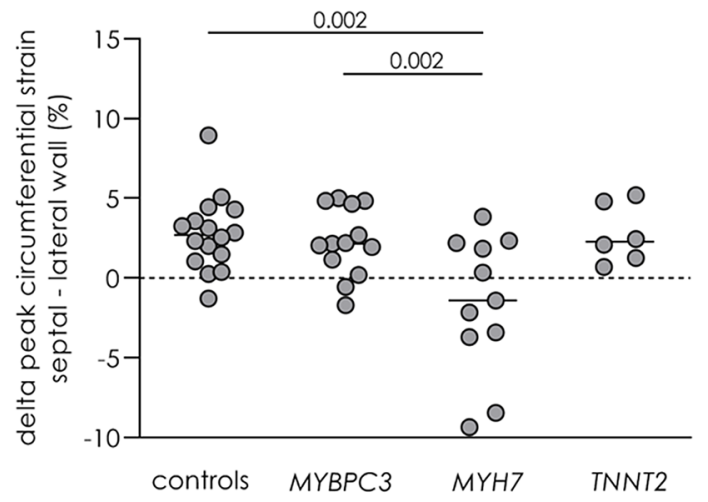

Fig. 3 Regional functional differences between gene variant carriers and controls. a The mean of peak circumferential strain of two septal or lateral segments at the basal and midventricular level, and $\mathbf{b}$ the

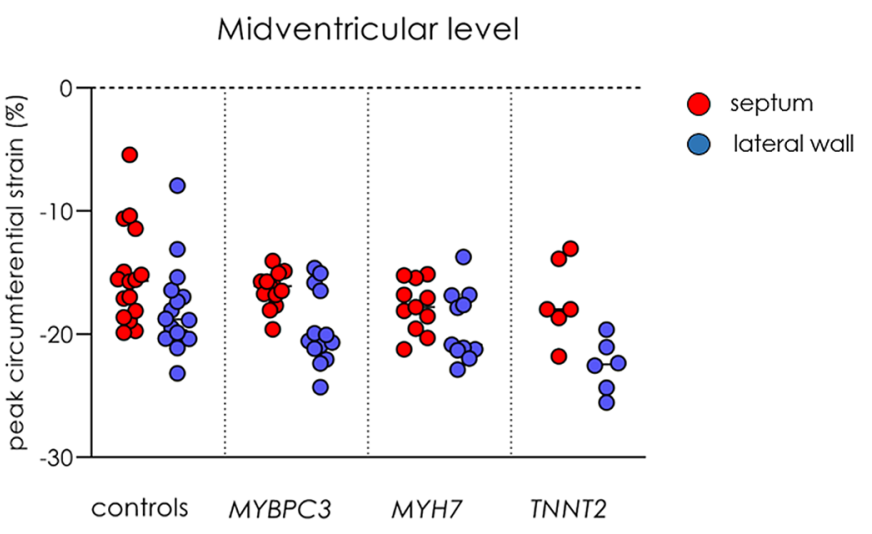

mean of the difference in peak circumferential strain between septum and lateral wall at basal and midventricular level. Data are presented as mean with standard deviation.* $\mathrm{p}<0.025$ septum vs. lateral wall 


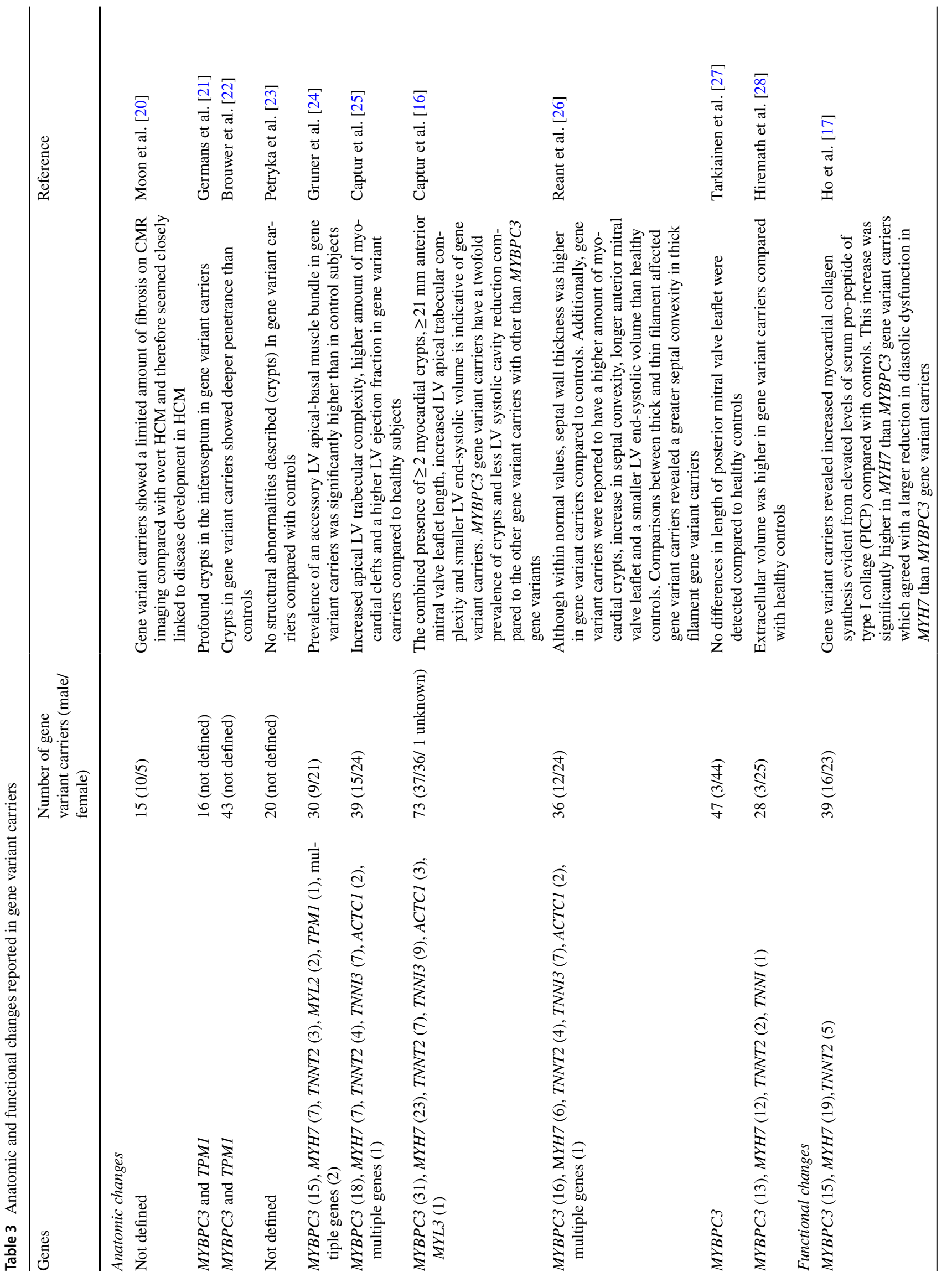




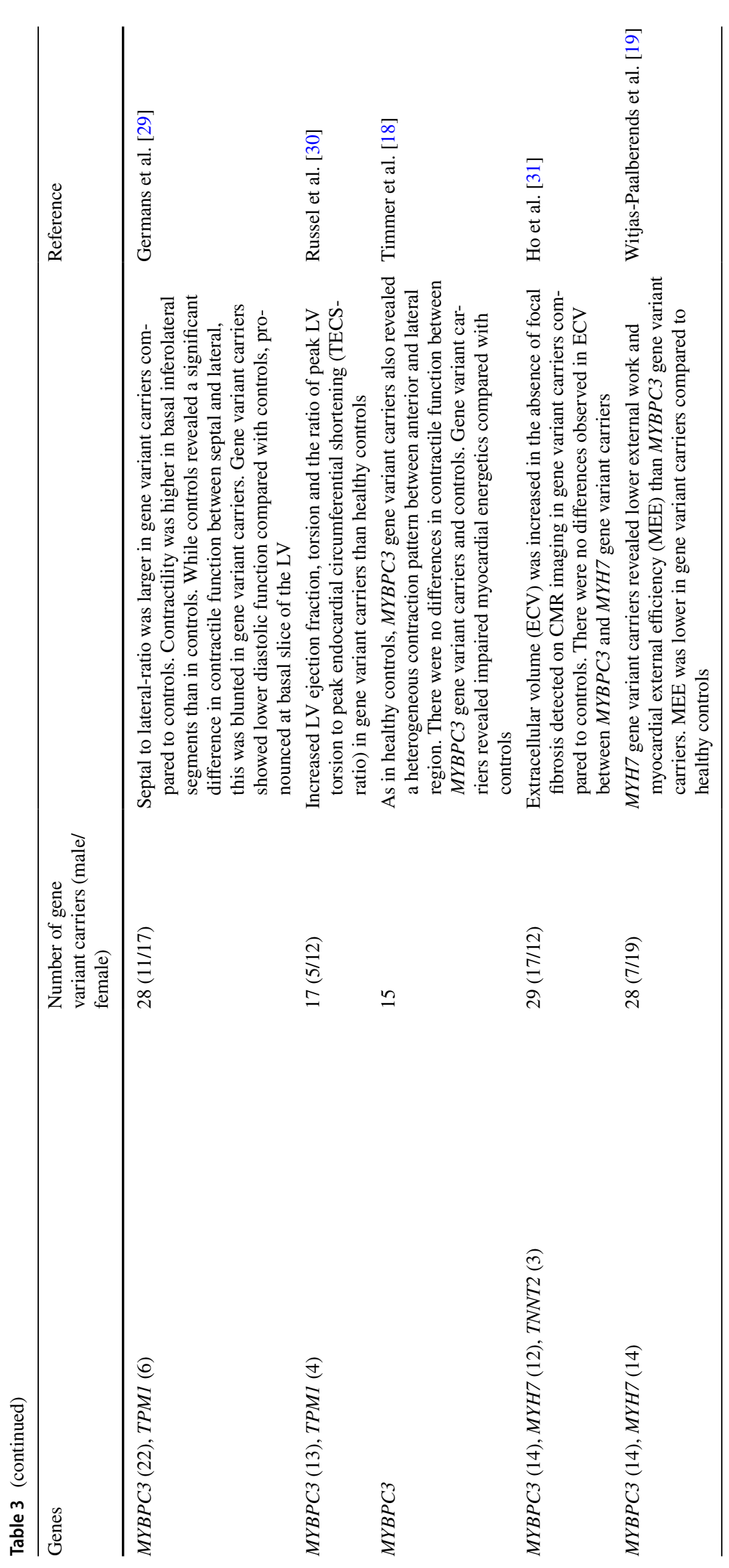




\section{Discussion}

Our case-control study in preclinical female variant carriers using state-of-the-art cardiac imaging shows a subtle change in cardiac function only in individuals with a MYH7 gene variant. Previous studies reported myocardial alterations in asymptomatic gene variant carriers, although these studies did not specify genotype or sex (summarized in Table 3) [16-31]. These alterations include differences in anatomical and functional level, such as as the amount fibrotic tissue or number of clefts in the myocardium and different length of the anterior mitral valve leaflet [16, 20-28], and a higher LV ejection fraction and torsion and altered myocardial metabolism [17-19, 29-31].

Strain measurements in asymptomatic carriers harboring thick filament gene variants, demonstrated comparable global and regional systolic strain as observerd in healthy controls [32]. A sub-analysis in the latter study comparing 35 MYH7 with 24 MYBPC3 gene variant carriers revealed a younger study population and higher peak longitudinal strain in $M Y H 7$ compared to $M Y B P C 3$ gene variant carriers [32]. While Ho et al. have not specified the mechanism in the higher global longitudinal stain in $\mathrm{MYH7}$ gene carriers [32], it may be speculated that a higher systolic strain at a regional level may explain this observation.

A previous study from our group investigating the effect of thick and thin filament gene variants $(M Y H 7$ and $M Y B P C 3$ ) associated with $\mathrm{HCM}$ on human cardiac myofilament function, demonstrated significantly higher tension cost, i.e. the amount of energy used during force development, in $M Y H 7$ compared to MYBPC3 [19], which coincided with a larger reduction in in vivo myocardial external efficiency compared to the control group in $M Y H 7$ than in MYBPC3 carriers. Follow-up studies showed that the reduction in myocardial external efficiency is present in individuals with thick and thin filament gene variants, and is explained by an increased cardiac oxygen consumption rather than altered contractile properties $[33,34]$. The present study shows a subtle change in the contraction pattern in preclinical female $M Y H 7$ carriers, which was not seen in $M Y B P C 3$ carriers. While this subtle change in MYH7 carriers may in part explain the gene-specific difference in cardiac efficiency [19], the current and previous studies [20,34] indicate that changes in energy consumption (i.e. increased oxygen consumption) rather than perturbations in (regional) contractile properties of the heart muscle characterize the very early disease stage of HCM.

With respect to gene variant-specific in vitro findings, functional properties of sarcomeres affected by thickfilament gene variants, obtained from tissue of HCM patients who underwent septal myectomy, revealed lower maximal force production in cardiac muscle strips containing $M Y H 7$ gene variants than in tissue with $M Y B P C 3$ gene variants [19]. Additionally, compared to tissue from genotype mutation-negative HCM patients, $M Y H 7$ affected sarcomere gene variants revealed increased kinetics of tension development $[35,36]$. Also, as HCM is most frequently inherited through a heterogeneous manner, allelic transcription, which occurs in a stochastic manner, may lead to variable expression of healthy and mutant proteins $[37,38]$ and may cause inhomogeneous contraction and relaxation. On in vivo cardiac imaging, this may lead to an increase in regional circumferential strain as seen in our study cohort and longitudinal strain observed in previous work [32]. However, the step from in vitro sarcomere function to in vivo circumferential strain imaging may be too large, since the effect of extracellular volume in the myocardium and myofiber disarray are challenging to take into account in in vitro experiments.

\section{Limitations}

The number of recruited carriers were limited, therefore, very subtle functional differences may have remained undetected. However, myocardial tissue tagging is a robust and sensitive method to evaluate regional function, and therefore the clinical value of subtle differences not detected with the method with these number of carriers is limited. In addition, controls were not genotyped. There is a small chance that within this group op controls, unidentified carriers were present.

\section{Conclusions}

Overall, CMR combined with tissue tagging detects subtle gene-specific regional differences in contractility. However, assessment of regional contraction by CMR tissue tagging currently does not aid in the identification of early cardiac disease changes in the preclinical genotype-positive population. Moreover, our study shows that there are no major contractile deficits in asymptomatic females carrying a pathogenic gene variant, which would justify the use of CMR imaging for earlier diagnosis.

Acknowledgments We thank Peter van der Ven for his statistical analysis.

Funding We acknowledge support from the Netherlands Cardiovascular Research Initiative: An initiative supported by the Dutch Heart Foundation, CVON2014-40 DOSIS and NWO (VICI 91818602).

\section{Compliance with ethical standards}

Conflicts of interest The authors declare that they have no conflict of interest. 
Open Access This article is licensed under a Creative Commons Attribution 4.0 International License, which permits use, sharing, adaptation, distribution and reproduction in any medium or format, as long as you give appropriate credit to the original author(s) and the source, provide a link to the Creative Commons licence, and indicate if changes were made. The images or other third party material in this article are included in the article's Creative Commons licence, unless indicated otherwise in a credit line to the material. If material is not included in the article's Creative Commons licence and your intended use is not permitted by statutory regulation or exceeds the permitted use, you will need to obtain permission directly from the copyright holder. To view a copy of this licence, visit http://creativecommons.org/licenses/by/4.0/.

\section{References}

1. Semsarian C et al (2015) New perspectives on the prevalence of hypertrophic cardiomyopathy. J Am Coll Cardiol 65(12):1249-1254

2. Elliott PM et al (2014) 2014 ESC Guidelines on diagnosis and management of hypertrophic cardiomyopathy: the Task Force for the Diagnosis and Management of Hypertrophic Cardiomyopathy of the European Society of Cardiology (ESC). Eur Heart J 35(39):2733-2779

3. Ho CY et al (2015) Genetic advances in sarcomeric cardiomyopathies: state of the art. Cardiovasc Res 105(4):397-408

4. Girolami F et al (2006) A molecular screening strategy based on beta-myosin heavy chain, cardiac myosin binding protein $\mathrm{C}$ and troponin $\mathrm{T}$ genes in Italian patients with hypertrophic cardiomyopathy. J Cardiovasc Med (Hagerstown) 7(8):601-607

5. Nijenkamp L et al (2018) Sex differences at the time of myectomy in hypertrophic cardiomyopathy. Circ Heart Fail 11(6):e004133

6. Huurman R et al (2020) Effect of body surface area and gender on wall thickness thresholds in hypertrophic cardiomyopathy. Neth Heart J 28(1):37-43

7. van Driel B et al (2019) Sex differences in hypertrophic cardiomyopathy: new insights. Curr Opin Cardiol 34(3):254-259

8. Olivotto I et al (2005) Gender-related differences in the clinical presentation and outcome of hypertrophic cardiomyopathy. J Am Coll Cardiol 46(3):480-487

9. Kubo $\mathrm{T}$ et al (2010) Gender-specific differences in the clinical features of hypertrophic cardiomyopathy in a community-based Japanese population: results from Kochi RYOMA study. J Cardiol 56(3):314-319

10. Marstrand P et al (2020) Hypertrophic cardiomyopathy with left ventricular systolic dysfunction: insights from the SHaRe Registry. Circulation 141(17):1371-1383

11. Marcus JT et al (1999) The influence of through-plane motion on left ventricular volumes measured by magnetic resonance imaging: implications for image acquisition and analysis. J Cardiovasc Magn Reson 1(1):1-6

12. Zwanenburg JJ et al (2003) Steady-state free precession with myocardial tagging: CSPAMM in a single breathhold. Magn Reson Med 49(4):722-730

13. Creatis, inTag, CMRSegTools and CMRDiffTools. A suite of tools for analysing cardiac MR images

14. Swoboda PP et al (2014) Reproducibility of myocardial strain and left ventricular twist measured using complementary spatial modulation of magnetization. J Magn Reson Imaging 39(4):887-894

15. Cerqueira MD et al (2002) Standardized myocardial segmentation and nomenclature for tomographic imaging of the heart. A statement for healthcare professionals from the Cardiac Imaging Committee of the Council on Clinical Cardiology of the American Heart Association. Circulation 105(4):539-542
16. Captur G et al (2014) Prediction of sarcomere mutations in subclinical hypertrophic cardiomyopathy. Circ Cardiovasc Imaging 7(6):863-871

17. Ho CY et al (2010) Myocardial fibrosis as an early manifestation of hypertrophic cardiomyopathy. N Engl J Med 363(6):552-563

18. Timmer SA et al (2011) Carriers of the hypertrophic cardiomyopathy MYBPC 3 mutation are characterized by reduced myocardial efficiency in the absence of hypertrophy and microvascular dysfunction. Eur J Heart Fail 13(12):1283-1289

19. Witjas-Paalberends ER et al (2014) Gene-specific increase in the energetic cost of contraction in hypertrophic cardiomyopathy caused by thick filament mutations. Cardiovasc Res 103(2):248-257

20. Moon JC et al (2005) Myocardial late gadolinium enhancement cardiovascular magnetic resonance in hypertrophic cardiomyopathy caused by mutations in troponin I. Heart 91(8):1036-1040

21. Germans $\mathrm{T}$ et al (2006) Structural abnormalities of the inferoseptal left ventricular wall detected by cardiac magnetic resonance imaging in carriers of hypertrophic cardiomyopathy mutations. J Am Coll Cardiol 48(12):2518-2523

22. Brouwer WP et al (2012) Multiple myocardial crypts on modified long-axis view are a specific finding in pre-hypertrophic HCM mutation carriers. Eur Heart J Cardiovasc Imaging 13(4):292-297

23. Petryka $\mathbf{J}$ et al (2014) Prevalence of inferobasal myocardial crypts among patients referred for cardiovascular magnetic resonance. Circ Cardiovasc Imaging 7(2):259-264

24. Gruner $C$ et al (2014) Significance of left ventricular apical-basal muscle bundle identified by cardiovascular magnetic resonance imaging in patients with hypertrophic cardiomyopathy. Eur Heart J 35(39):2706-2713

25. Captur $\mathrm{G}$ et al (2014) Abnormal cardiac formation in hypertrophic cardiomyopathy: fractal analysis of trabeculae and preclinical gene expression. Circ Cardiovasc Genet 7(3):241-248

26. Reant $P$ et al (2015) Abnormal septal convexity into the left ventricle occurs in subclinical hypertrophic cardiomyopathy. J Cardiovasc Magn Reson 17:64

27. Tarkiainen $\mathrm{M}$ et al (2016) Cardiovascular magnetic resonance of mitral valve length in hypertrophic cardiomyopathy. J Cardiovasc Magn Reson 18(1):33

28. Hiremath $P$ et al (2016) Ultrasonic assessment of myocardial microstructure in hypertrophic cardiomyopathy sarcomere mutation carriers with and without left ventricular hypertrophy. Circ Heart Fail 9(9):e003026

29. Germans $\mathrm{T}$ et al (2010) How do hypertrophic cardiomyopathy mutations affect myocardial function in carriers with normal wall thickness? Assessment with cardiovascular magnetic resonance. J Cardiovasc Magn Reson 12:13

30. Russel IK et al (2011) Increased left ventricular torsion in hypertrophic cardiomyopathy mutation carriers with normal wall thickness. J Cardiovasc Magn Reson 13:3

31. Ho CY et al (2013) T1 measurements identify extracellular volume expansion in hypertrophic cardiomyopathy sarcomere mutation carriers with and without left ventricular hypertrophy. Circ Cardiovasc Imaging 6(3):415-422

32. Ho CY et al (2009) Echocardiographic strain imaging to assess early and late consequences of sarcomere mutations in hypertrophic cardiomyopathy. Circ Cardiovasc Genet 2(4):314-321

33. Guclu A et al (2017) Disease stage-dependent changes in cardiac contractile performance and oxygen utilization underlie reduced myocardial efficiency in human inherited hypertrophic cardiomyopathy. Circ Cardiovasc Imaging 10(5):e005604

34. Parbhudayal RY et al (2020) Increased myocardial oxygen consumption precedes contractile dysfunction in hypertrophic cardiomyopathy caused by pathogenic TNNT2 gene variants. J Am Heart Assoc 9(8):e015316 
35. Tyska MJ et al (2000) Single-molecule mechanics of R403Q cardiac myosin isolated from the mouse model of familial hypertrophic cardiomyopathy. Circ Res 86(7):737-744

36. Belus A et al (2008) The familial hypertrophic cardiomyopathyassociated myosin mutation R403Q accelerates tension generation and relaxation of human cardiac myofibrils. J Physiol 586(15):3639-3644

37. Kraft $\mathrm{T}$ et al (2016) Hypertrophic cardiomyopathy: cell-to-cell imbalance in gene expression and contraction force as trigger for disease phenotype development. Circ Res 119(9):992-995
38. Parbhudayal RY et al (2018) Variable cardiac myosin binding protein-C expression in the myofilaments due to MYBPC3 mutations in hypertrophic cardiomyopathy. J Mol Cell Cardiol 123:59-63

Publisher's Note Springer Nature remains neutral with regard to jurisdictional claims in published maps and institutional affiliations. 